\title{
Experience of Treating Acute Appendicitis in Tashkent
}

\author{
Akilov Khabibullo Ataullayevich, Urmanov Nozim Tursinaliyevich, Primov Farhod Sharifjonovich, \\ Djurayev Jurat Atkhamovich*, Yuldashev Tolib Akromovich, \\ Khidoyatov Komronbek Zaynitdinovich
}

Republican Research Centre of Emergency Medicine of the Health Ministry of the Republic of Uzbekistan, Tashkent, Republic of Uzbekistan

\author{
Email address: \\ uzmedicine@mail.ru (D. J. Atkhamovich) \\ ${ }^{*}$ Corresponding author
}

\section{To cite this article:}

Akilov Khabibullo Ataullayevich, Urmanov Nozim Tursinaliyevich, Primov Farhod Sharifjonovich, Djurayev Jurat Atkhamovich, Yuldashev Tolib Akromovich, Khidoyatov Komronbek Zaynitdinovich. Experience of Treating Acute Appendicitis in Tashkent. American Journal of Pediatrics. Vol. 6, No. 1, 2020, pp. 68-72. doi: 10.11648/j.ajp.20200601.22

Received: January 20, 2020; Accepted: February 17, 2020; Published: February 28, 2020

\begin{abstract}
Modern diagnostics of acute appendicitis in children should include abdominal ultrasound investigation upon admission to a specialized hospital, if necessary, in an ultrasound monitoring mode during clinical dynamic observation of the child. Surgical treatment is indicated only for destructive appendicitis. The method of a surgical treatment choice is laparoscopic surgery including destructive appendicitis complicated by appendicular peritonitis. Surgeries in acute appendicitis make up $75 \%$ of all emergency surgical interventions. Annually only in the Republican Research Centre of Emergency Medicine 700 appendectomies in children are made on average. The article summarizes data obtained in the retrospective analysis of case histories of 6256 patients with acute appendicitis and its complications who were hospitalized in the pediatric department of emergency surgery for 12 years. The following incidence of acute appendicitis in children is reported: from 1 to 3 years old - 0.6 per 1000 ; from 4 to $7-1.4$ - 2.6 per $1000 ; 13$ years of age -8 per 1000 . The authors discuss specific features in the clinical picture of acute appendicitis in children depending on patient's age and anatomical location of the appendix. The clinical picture in children of older age and of the first three years of life is discussed separately. Among 6256 pediatric patients with acute appendicitis $72(1.15 \%)$ were children younger than 3 years. The authors have substantiated a complex of diagnostic and therapeutic manipulations as well as tactic options to the treatment of this category of patients. Widespread implementation of the laparoscopic technique into surgical practice has significantly changed the tactics of treatment. The number of surgeries for simple appendicitis in children has decreased up to $3.9-7 \%$.
\end{abstract}

Keywords: Acute Appendicitis, Appendectomy, Laparoscopy, Surgery, Pediatric Surgery

\section{Introduction}

Acute appendicitis in children as well as in adults is the most common surgical disease requiring emergency surgery. Surgeries in acute appendicitis make up $75 \%$ of all emergency surgical interventions. Annually only in the Republican Research Centre of Emergency Medicine 700 appendectomies in children are made on average. The morbidity of acute appendicitis: from 1 to 3 years old - 0.6 per 1000; from 4 to 7 years - $1.4-2.6$ per $1000 ; 13$ years old 8 per 1000 children $[1,2]$.

Timely and correct diagnostics is the key to successful treatment of surgical diseases in children. Quite often, due to the anatomical and physiological characteristics of children (the most difficult age is up to 3 years), the diagnostics of acute appendicitis in children, especially at an early age, causes difficulties even for qualified pediatric surgeons [3]. It is important for surgeons to be able to distinguish acute appendicitis from a number of non-surgical diseases of childhood, often simulating a picture of acute appendicitis. Conversely, inflammation of the appendix can initially appear as a mild disease, and the child, in this case, gets to the surgeon already in a terminal state. The ability to differentiate the disease is an indispensable condition for making the correct diagnosis, preventing complications and achieving the ultimate goal - the child's recovery $[2,4]$. 
Undoubtedly, the ever wider introduction of laparoscopy into practice allowed to raise the diagnostics of acute appendicitis to a qualitatively new level. The number of surgeries due to simple (non-destructive) appendicitis in children decreased to $3.9-7 \%[1,2,5,6]$ and in some clinics with the greatest experience up to $0.6-2.8 \%$ and even lower [7-10]. An analysis of the literature shows that the number of children operated by pediatric surgeons (in departments that did not use the laparoscopic diagnostic method) due to simple (nondestructive) appendicitis is $30.3-38.7 \%$, reaching $42.3 \%$ in children under the age of 3 years. Even in the leading clinics of the world, before the use of laparoscopy, these indicators were at the level of $30-36 \%[4,11]$.

The overdiagnosisof acute appendicitis by doctors of various specialties can be considered justified only at the pre-hospital stage. It should be completely agree with the opinion of M. R. Rokitsky that it's better to send 10 children with suspected acute appendicitis to the surgeon in vain than to miss this disease in one of them. Fear of aoverdiagnostic error and the probability of developing perforated peritonitis leads the doctor tooverdiagnosis. However, this tactic should be considered justified only at the final stage of complex cases of diagnostics and in no way as the basic rule of treatment for children with abdominal pain. Moreover, in doubtful cases, the surgery must be preceded by a period of dynamic monitoring of the child during which a set of necessary diagnostic examinations is carried out- it allows to clarify the diagnostics and to avoid unnecessary surgeries. The time after which, in doubtful cases, surgical intervention in the departments of pediatric surgery should be applied is different and ranges from 6-12 to 24 hours [4, 11]. The literature data show that tactics based simply on overdiagnosisof a disease also do not save from mistakes. Consequently, the existing tactics of diagnosing appendicitis in children hospitalized in the department requires being reviewed. A number of diseases may be hidden behind the pain syndrome and they require intensive and sometimes emergency treatment [3, 5, 11, 12]. Research results of $\mathrm{Yu}$. F. Isakov et al. allowed to establish that in $40.3 \%$ of children operated on with a diagnosis of catarrhal or secondary altered appendicitis, other diseases simulating acute appendicitis were observed. Thus, performing vain surgeries against the background of an undiagnosed main disease accompanied by abdominal pain, can aggravate the severity of a sick child condition and is fraught with the development of complications, and in some cases can lead to death $[7,13]$.

Thus, the analysis of the literature shows that there is an urgent need for a detailed examination of the diagnostic and therapeutic tactics adopted in hospitals that provide emergency surgical care to the children's population.

Aimof the research is to study the main causes of late visits based on a retrospective analysis as well as errors in the diagnostics and surgical tactics of acute appendicitis in children.

\section{Material and Methods}

The work has been based on a retrospective analysis of the medical history of 6256 patients with a diagnosis of acute appendicitis and its complications who were hospitalized in the emergency department of pediatric surgery for the period from 2005 to 2017 . There were 3855 boys $(61.1 \%)$ and 2391 girls $(38.1 \%)$. There was a following age rate among children: infants ( $0-3$ years $)$ - $72(1.15 \%)$ cases, younger age (4-7 years) - 1200 (19.1\%), school age (8-14 years) - 3525 (56.3\%), adolescents (15-18 years) - 1459 (23.3\%) patients. By the nature of morphological changes, 1185 (18.9\%) children had simple appendicitis, in 3145 (50.2\%) cases we observed a phlegmonous form, 789 (12.6\%) children had gangrenous appendicitis, and 1137 (18.1\%) patients complicated forms: appendicular infiltrate in $15(0.2 \%)$, local limited peritonitis (appendicular abscess) in $122(1.9 \%)$, local unlimited peritonitis in $386(6.1 \%)$, diffuse peritonitis in 378 $(6.0 \%)$ and in $236(3.7 \%)$ - diffuse peritonitis. According to the clinical course, $1564(25.0 \%)$ patients had an atypical course of the disease and $4692(75.0 \%)$ had a typical course. $3100(49.5 \%)$ of all 6256 patients went to the doctor on their own, $2900(46.3 \%)$ - by ambulance, $256(4.2 \%)$ patients were transferred from other institutions. According to delivery time, children were divided into the following groups: up to 6 hours - $980(15.6 \%)$ children, from 6 to 12 hours - 2800 (44.7\%), from 12 to 24 hours $-1376(21.9 \%)$, more than 24 hours - $1100(17.5 \%)$ patients.

\section{Results and Discussion}

For making a diagnosis all patients with a clinic of acute appendicitis and with suspected acute appendicitis were hospitalized in the departments of pediatric surgery and examined according to the standard. Clinical manifestations of acute appendicitis in children are variable and largely depend on the reactivity of the organism, anatomical position of the appendix and the child's age. A general characteristic of the disease clinical picture is the prevalence of general non-specific symptoms over local ones. The relative immaturity of individual organs and systems in the genesis of such reactions, primarily the central nervous system and the autonomic nervous system, is important. The younger the child, the more these manifestations are evident.

The difficulties of diagnosis are also exacerbated by the difficulties of examining children and identifying objective local signs of the disease. Their reliability depends on many factors (contact with the child, knowledge of the examination method, individual experience of the doctor, etc.). The influence of these factors is especially evident in the recognition of acute appendicitis in children of early age. In connection with these circumstances, we also decided that it was necessary to separately consider the clinical picture in children of the first 3 years of life and in elder children. There were $72(1.15 \%)$ of 6256 children under 3 years in our observations. The clinical picture of acute appendicitis in children of early age depends not only on the reactivity of the 
child's body, but also on the age-related anatomical and physiological features. Firstly, due to the functional immaturity of the nervous system at this age, almost all acute inflammatory diseases have a similar clinical picture (high body temperature, repeated vomiting, intestinal function failure). Secondly, the inflammatory process in the appendix proceeds extremely rapidly in children. Thirdly, there are specific difficulties in examining children of early age.

There were no direct indications of abdominal pain in this group of children; the presence of this symptom was judged only by indirect signs. It was known from the anamnesis that in more than $75 \%$ of cases parents noted lethargy, moodiness, low contact of the child, fever, often to $38-39^{\circ} \mathrm{C}$ (in 95\%). A fairly constant symptom was repeated vomiting up to $3-5$ times $(85 \%)$ which refers to the peculiarities of the disease course at this age. In almost $25 \%$ of cases loose stools were noted, this fact can lead to an erroneous diagnosis of firstlevel doctors. Twenty $(0.31 \%)$ children of $72(1.15 \%)$ patients of early age with acute appendicitis (it should be noted that in our observations the youngest child was 3 months old) already admitted with peritonitis clinic picture. They, as often happens, have been delivered to the clinic at a later date from the onset of the disease. The main reason for the late admission is the lack of a typical clinical picture and insufficient knowledge of acute appendicitis clinic in children of early age by first-level doctors. At objective examination the condition of children was serious due to the clinic of endotoxicosis. According to the severity of the condition, the children were hospitalized to the ICU department where in parallel with the examination, preoperative preparation was carried out: a general analysis of blood, urine, standing fluoroscopy, ultrasound investigation. In the analyzes of peripheral blood in children with peritonitis in $98 \%$ of cases leukocytosis was determined with a predominance of neutrophils, reaching 10-20 $109 / 1$, and an increase of ESR. A typical symptom for children of early age with peritonitis is multiple horizontal small intestinal levels or free gas under the diaphragm on an overview radiography of the abdominal cavity. On ultrasound investigation - free fluid between the loops of the intestines and the extended loops of the small intestine. In $33(0.52 \%)$ cases children of early age with acute appendicitis were delivered within 12 hours from the onset of the disease. The diagnosis was confirmed at the initial examination in these patients. In the remaining $19(0.33 \%)$ cases the children were hospitalized to the departments with suspected acute appendicitis. During the dynamic observation the patients were examined in a dream: a symptom of a repulsive arm was typical, at repeated analyzes an increase in leukocytes with a shift was noted; rectal examinations were carried out, in addition - fluoroscopy and ultrasound investigation. The diagnosis was confirmed by dynamic observation. This group of patients had an atypical location of the appendix, which also led to the late diagnostics of acute appendicitis.

There were $6214(85.6 \%)$ children over 3 years old from of the total number of patients. More than 2000 children admitted later than 12 hours, the reason was atypical forms of acute appendicitis and errors in diagnostics by first-level doctors, widespread use of non-steroidal anti-inflammatory drugs by parents for pain in the abdomen in children. In this group (older than 3 years), the disease began gradually and, as a rule, had a typical clinical picture. The main symptom was abdominal pain observed in $100 \%$ of children. Abdominal pain in older children occurred in the epigastric region or near the navel, then captured the entire abdomen and only a few hours later localized in a certain place, most often in the right iliac region, Kocher - Volkovich symptom was observed in 3450 (47.5\%) children, mostly in older ones. The pain is usually constant aching in nature. Unfortunately, surgeons are deprived of the ability to objectively determine the intensity of pain in children and judge it only by the reaction of the patient. Some children cry not because of sharp pains at all, while others, fearing the operation, hide their presence. Before the examination, having talked with the child, the surgeon must distract his attention from the disease, enter into confidence in him. In addition, the doctor has to take into account indirect manifestations of pain - how the child slept, what was his behavior during the day and etc. Vomiting and nausea being frequent, but not constant symptoms of acute appendicitis were observed in 4890 (78.1\%) children, more often in children of younger age and with a complicated course of the disease. Vomiting is observed mainly in the first hours of the disease and most often is single. Repeated vomiting is a sign of increasing intoxication. The tongue is lightly coated in white, moist. In 2450 (39.1\%) cases, stool retention was observed, and in 879 $(14.0 \%)$ - loose, frequent stool with an admixture of mucus and it led to diagnostic errors. Most often there is a violation of appetite, children refuse to eat. The body temperature of $2890(46.1 \%)$ older children in the first hours of the disease was normal or subfebrile. The pulse becomes more frequent as the inflammatory process develops. Tachycardia which does not correspond to the height of the body temperature is typical for children with inflammatory processes in the abdominal cavity, which was detected in 2600 (41.5\%) children. The discrepancy between heart rate and temperature often helps in the diagnosis, when other symptoms are erased or the child hides the pain. The general condition of children with acute appendicitis may be satisfactory, however, it worsens with an increase of inflammatory phenomena, their spread to the peritoneum or the appearance of intoxication. Patients with complicated peritonitis were usually in a forced position, lying on their backs or right side with legs bent and raised to the abdomen. Most often when viewed, they do not reveal changes in the shape of the abdomen. The abdomen is involved in the act of breathing in the initial stages of the disease. A palpation examination of the abdomen should begin with the left, healthy half, first superficially, one of the main objective symptoms is revealed - muscle tension of the anterior abdominal wall, which in acute appendicitis is more often in the right half of the abdomen or it is more evident on the right than on the left. Simultaneous superficial palpation of the abdomen on both sides is performed for a better determination of muscle tension. Moreover, the possibility of 
establishing a varying degree of the rectus muscles tension is significantly increased. Painfulness when palpating the abdominal wall is usually localized in the right iliac region and is already determined in the first hours of the disease. The intensity of pain varies and depends both on the depth of the destructive process, and on the particular reaction of the child to palpation. In this regard, the symptom of pain should be treated with caution. A very active reaction of the child and crying during palpation of the abdomen often causes unreasonable surgery, and, conversely, very slight pain or lack of it during deep palpation can cause the surgeon to refuse the necessary intervention. Examining a child with suspected acute appendicitis, it is a mistake to look for the so-called pain points and check for numerous pain symptoms typical for acute appendicitis in adults. It is difficult to reliably identify these symptoms in a child and hence of their value is very doubtful. However, in older children, the Shchetkin - Blumberg symptom has a certain diagnostic value. Pain in the right iliac region is noted with percussion of the abdominal wall. A digital perrectum test is performed in children with suspected acute appendicitis. In this case, overhanging, infiltration in the abdominal cavity, pain, sponginess of rectal wall, enlarged pararectal lymph nodes, and in girls, in addition, changes of the internal genital organs can be detected. Laboratory data of acute appendicitis are not specific and can only indicate the presence of an inflammatory process. Changes in the blood are expressed in an increase in the number of leukocytes to 10-12 $109 / 1$, rarely to $13-15 \cdot 109 / 1$. The leukocyte formula is of great importance for assessing the severity of the inflammatory process: left shift, increase in the number of stab neutrophils; the appearance of young forms and even myelocytes can be observed in children with destructive changes in the appendix. ESR in the first hours of the disease usually remains unchanged and only in advanced cases, with late admission and the development of complications it increases. Changes are usually not observed in urine at acute appendicitis. They are detected only with pronounced intoxication and are characterized by the appearance of protein, red blood cells and hyaline cylinders. In addition, pathological changes in urine may appear with a pelvic location of the appendix. Emergency surgical intervention immediately after hospitalization in a surgical hospital or after short-term preoperative preparation is indicated when a diagnosis of acute appendicitis is established, impossibility of its exclusion after the entire complex of diagnostic measures and dynamic observation for no more than 12 hours.

Anesthesia in children, especially at an early age, should be only general. An important point preceding anesthesia is the psychological preparation of the patient. $6241(99.7 \%)$ of $6256(100 \%)$ patients with acute appendicitis were performed emergency surgeries. When diagnosing, $0.3 \%$ of children received conservative therapy with subsequent interval appendectomy. $3800(60.5 \%)$ of 6241 (99.7\%) operated patients were performed surgery after an initial examination within one hour, 1200 (19.1\%) - after short-term preoperative preparation within 3 hours. 600 (9.5\%) hospitalized children with suspected acute appendicitis were operated on within 12 hours, because the diagnosis was confirmed during dynamic observation. $641(10.2 \%)$ patients were performed surgery within 24 hours: acute appendicitis was excluded by the onduty team in these children, but after examination by the hospital doctors, the diagnosis of acute appendicitis was established and surgical intervention was recommended. Surgical tactics of acute appendicitis in children is fundamentally little different from that in adults. However, there are features that are most evident in the first years of a child's life at the surgical treatment of various forms of acute appendicitis.

Our clinic has successfully performed laparoscopic appendectomy for recent years. It has undeniable advantages such as a lower incidence of postoperative complications and a good cosmetic effect. 1988 (31.7\%) of 6241 operated patients were performed a laparoscopic appendectomy. This number included children with suspected acute appendicitis, girls of pubertal age, children with increased nutrition and with a clinic of uncomplicated appendicitis predominated, 78 (1.2\%) switched to conversion. A traditional appendectomy with a McBurney's incision was performed in 3639 (58.1\%) children, in $644(10.2 \%)$ cases with complicated forms of the disease laparotomy surgery was performed. Antibiotic therapy was performed in the postoperative period. As a rule, at a simple and phlegmonous form of appendicitis a single interoperative antibiotic therapy was carried out with cephalosporins of the 1st - 2nd generation, at complicated forms until the response of bacteriological inoculation - with 3rd-generation cephalosporins + metronidozole. Anesthesia after traditional appendectomy has been carried out for 2-3 days, after laparoscopic surgery - usually within 1 day. On the 4th -5 th day children after traditional appendectomy and on the 2nd 3rd after laparoscopic appendectomy were discharged home for further outpatient treatment. There were no fatal outcomes.

\section{Conclusion}

Thus, acute appendicitis is the most common childhood surgical disease requiring emergency surgery. Usually the first person to meet with a sick child is a pediatrician, on whom the prevention of acute appendicitis complicated forms, mainly due to late diagnosis, largely depends. The immaturity of the structures and functional systems of a growing organism leads to a variety of reactions, and consequently, to clinical manifestations in response to the arising pathological process. This, in turn, determines diagnostic difficulties, especially in the early stages of the disease. One of the approaches to reducing the number of possible miscalculations and errors can be mastering the principle of multi-stage diagnostics: initial diagnosis during a physical examination of a child, an updated diagnosis after laboratory and special studies, taking into account changes of the clinical picture during dynamic observation and, as a result of homeostasis correction, examining a child in a dream and assessment of the physical data obtained with it, analysis of operational information, monitoring of patients in 
the postoperative period, when a picture of complication becomes clear, and finally - a microscopic examination of the appendix.

\section{Conflict of Interest}

The authors declare no conflict of interest.

\section{Financing (Acknowledgements)}

The study did not have sponsorship.

\section{References}

[1] Abushkin I. A. Non-invasive diagnosis of delimited purulentinflammatory processes of the abdomen in children. In the book: Modern technologies in pediatrics and pediatric surgery. M.: 2012; 340 .

[2] Averin V. I. Averin, V. A. Katko, A. A. Svirsky Diagnostic and therapeutic laparoscopy in pediatric surgery. Actual issues of laparoscopy in pediatrics: Materials of the symposium. M.; 2014: 10-1.

[3] Alkhimovich V. N. Syndromic diagnostics in the prevention of errors in acute appendicitis in children. Bulletin of a general practitioner. $2000 ; 4: 12-8$.

[4] Abdullaev V. V., Fedenko G. V., Khodos E. G. et all. Diagnostic errors, complications, unforeseen situations when using traditional laparoscopy and video laparoscopy in emergency surgery. Endoscopic surgery. 2002; 5: 27-33.
[5] Sazhin V. P., Gorbich V. F., Alekseeva O. K. et all. Dynamics of nosocomial infections of the surgical department during the implementation of laparoscopic surgery. Endoscopic surgery. 2016; 5: 20.

[6] Abu-Yousef M. M., Bleacher J. J., Macer J. et al. High resolution sonography of acute appendicitis AJR. 2016; 149: $53-8$.

[7] IsakovYu. F., Stepanov E. A. Pediatric surgery on the threshold of the XXI century. Children's surgery. 2001; 4-7.

[8] Bendeck S. E., Nino-Murcia M., Berry G. J. et al. Imaging for suspected appendicitis: negative appendectomy and perforation rates. Radiology. 2012; 225 (1): 131-6.

[9] Bijnen C. L., Van Den Broek W. T., Bijnen A. B. et al Implications of removing a normal appendix. Dig. Surg. 2017; 20 (2): $115-21$.

[10] Hayden C. K., Kuchelmeister J., Lipscomb T. S., Sonography of acute appendicitis in childhood: Perforation versus nonperforation. Med. 2017; 11: 209-216.

[11] Helical C. T., Rao P. M., Rhea J. T., Novelline et al. technique for the diagnosis of appendicitis: Prospective evaluation of a focused appendix CT examination. Radiology. 2012; 202: 139-44.

[12] Holcomb G. W. Laparoscopic appendectomy in children. Lap. Surg. (Decker). 1993; 1 (3): 145-53.

[13] Horrow M. M. White D. S., Horrow J. C. Differentiation of perforated from nonperforated appendicitis at. Radiology. 2013; 227 (1): 46-51. 